\title{
Intrathoracic tuberculous lymphadenopathy: clinical and bronchoscopic features in 17 adults without parenchymal lesions
}

\author{
Reha Baran, Meltem Tor, Kemal Tahaoğlu, Kürşat Özvaran, Altan Kır, \\ Özkan Kızkın, Hatice Türker
}

\begin{abstract}
Background - Whilst intrathoracic lymphadenitis is a characteristic sign of primary tuberculosis in children, its presence without parenchymal lesions in adults is unusual and makes the diagnosis using noninvasive techniques difficult. The diagnostic role of bronchoscopy in adults with intrathoracic tuberculous lymphadenitis is reported.

Methods - Seventeen patients with intrathoracic lymphadenopathy seen during 1993 who had all undergone bronchoscopy and had been found to have tuberculosis in the absence of any parenchymal lung lesions were evaluated retrospectively.

Results - Right paratracheal lymphadenopathy was observed on the plain chest radiograph in all the patients. Fifteen of the 17 patients had an endobronchial abnormality and samples taken at bronchoscopy gave a definitive diagnosis in nine $(53 \%)$ of the 17 . Four patients had ulcerating endobronchial granuloma and all had biopsy samples positive for tuberculosis. Transbronchial or transcarinal needle aspiration samples were diagnostic in five of 11 patients (45\%) subjected to the procedure. Peripheral lymph node biopsy diagnosed tuberculosis in two cases and in the remaining six patients the diagnosis was achieved by mediastinoscopy or thoracotomy.

Conclusions - Bronchoscopy has an important role in the diagnosis of intrathoracic tuberculous lymphadenopathy in adults and should be considered before other invasive procedures.

(Thorax 1996;51:87-89)
\end{abstract}

Keywords: tuberculosis, mediastinal lymphadenopathy, diagnosis, bronchoscopy.

Although intrathoracic lymphadenitis is recognised as a characteristic manifestation of primary tuberculosis, especially in children, its presence in adults in the absence of any demonstrable parenchymal lesion presents a diagnostic problem. There is a low diagnostic yield from sputum examination in such patients. However, sputum negative cases can be further investigated by bronchoscopy which can be useful in patients with suspected parenchymal disease. ${ }^{1}$ The diagnostic role of bronchoscopy for intrathoracic lymphadenitis has not been extensively studied, ${ }^{2}$ so we report a retrospective study of the yield from bronchoscopy in 17 patients with intrathoracic lymphadenitis.

\section{Methods}

We retrospectively studied 17 adult patients (five women) of mean age $30 \cdot 1$ years (range 15-59 years) who presented during 1993 with intrathoracic lymphadenitis and no parenchymal lung lesion and were proven to have tuberculosis. The diagnosis of tuberculosis had been confirmed either by (1) a positive smear, (2) a positive culture of sputum, lymph node aspirate, or bronchial brushing, or (3) the presence of caseating granuloma in a bronchial or lymph node biopsy sample. Chest radiographs from all patients were assessed on admission and on subsequent follow up and all had undergone computed tomographic (CT) scanning of the thorax. All patients underwent a tuberculin skin test and had three sputum samples examined for acid fast bacilli and cultured on Loewenstein-Jensen medium.

Bronchoscopy had been undertaken on all patients either because of negative findings for acid fast bacilli on bacteriological examination (15 cases) or to rule out malignancy (two cases). Six patients in whom a diagnosis was not made from bronchoscopy then underwent intrathoracic lymph node biopsy by mediastinoscopy or thoracotomy. Bronchoscopy was performed under general anaesthesia with a rigid bronchoscope and bronchial secretions were taken for smear and culture. Transbronchial needle aspiration samples were obtained under radioscopic control using a wide bore needle.

\section{Results}

During the study period 6541 patients were treated for tuberculosis at our centre with only $17(0.26 \%)$ of these patients having intra-
18 April 1995

Revised version received

9 June 1995

Accepted for publication

14 September 1995

Pulmonary Diseases
R Baran
M Tor
K Tahaoğlu
K Özvaran
Ö Kuzkın
H Türker
Department of
Thoracic Surgery
A Kur
Sureyyapasa Center
for Chest Diseases and
Thoracic Surgery,
Maltepe,
85550 Istanbul, Turkey
Correspondence to:
Dr R Baran.
Received 7 February 1995
Returned to authors
18 April 1995
Revised version received
9 June 1995
Accepted for publication
14 September 1995




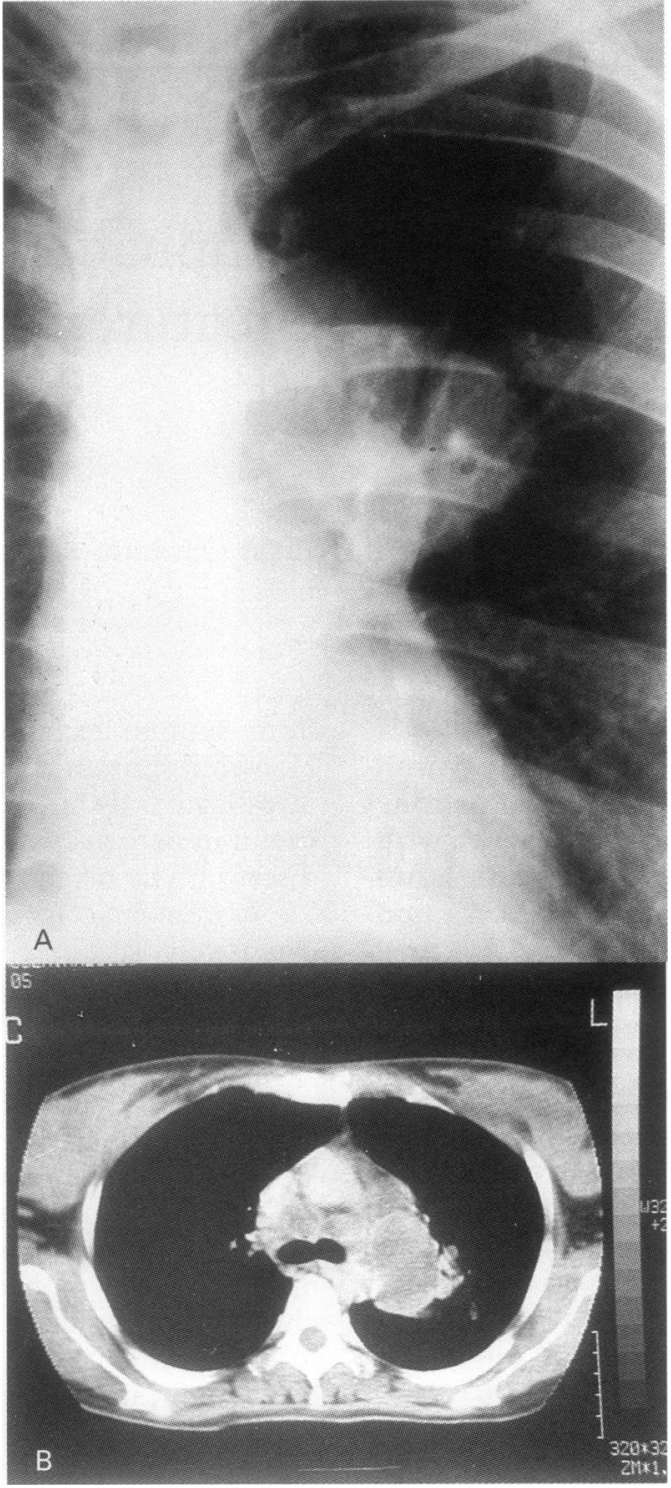

Figure 1 (A) A close up of the mediastinum and left hilum from a posteroanterior chest radiograph of a patient with bilateral mediastinal and left hilar lymph node involvement and $(B)$ computed tomographic scan of the same patient.

Table 1 Radiographic findings in 17 cases of intrathoracic tuberculous lymphadenopathy

\begin{tabular}{lll}
\hline $\begin{array}{l}\text { Paratracheal } \\
\text { lmphadenopathy }\end{array}$ & Hilar lymphadenopathy & No. of patients \\
\hline Bilateral & Bilateral & 3 \\
Bilateral & Right & 1 \\
Bilateral & Left & 2 \\
Bilateral & Right & 2 \\
Right & Bilateral & 1 \\
Right & - & 5 \\
Right & & \\
\hline
\end{tabular}

None of the patients had any concomitant pulmonary parenchymal lesion.

Table 2 Diagnosis of intrathoracic tuberculous lymphadenopathy

\begin{tabular}{lclc}
\hline Diagnostic method & No. examined & No. diagnosed & Positivity (\%) \\
\hline Peripheral lymph node (histological study) & 2 & 2 & 100 \\
Bronchoscopy & 17 & 9 & 53 \\
Mucosal biopsy for histological study & 4 & 4 & 100 \\
Transbronchial needle biopsy & 11 & 5 & $45 \cdot 4$ \\
Mediastinoscopy & 4 & 4 & 100 \\
Thoracotomy & 2 & 2 & 100 \\
\hline
\end{tabular}

thoracic lymphadenitis and no parenchymal lung lesion. Sputum was smear negative in all these 17 patients but was culture positive for Mycobacterium tuberculosis in three patients (18\%). Tuberculin skin test with PPD gave an induration of at least $10 \mathrm{~mm}$ in all cases (range 10-25 mm, median $16 \mathrm{~mm}$ ).

Right paratracheal nodes were seen radiographically in all patients. One patient had a small pleural effusion, eight patients had bilateral mediastinal lymph node involvement (fig $1 \mathrm{~A}$ and $1 \mathrm{~B}$ ) and 10 had hilar lymphadenopathy (table 1).

All but two patients had an endobronchial abnormality with a widened carina in eight patients, bronchial or tracheal displacement by lymph node enlargement in seven patients, ulcerating granuloma in four patients, and mucosal erythema and swelling in five patients. In all four patients with ulcerating granuloma bronchial biopsies revealed caseating granuloma and in three of these the biopsy cultures were positive for $M$ tuberculosis. Eleven patients underwent transbronchial or transcarinal needle aspiration and in five of these the samples yielded $M$ tuberculosis. Thus, bronchoscopy diagnosed tuberculosis in a total of nine $(53 \%)$ of the 17 patients.

In two patients with peripheral lymph node enlargement the diagnosis of tuberculosis was made by prior lymph node biopsy whilst the bronchoscopy was not diagnostic. In the remaining six patients the diagnosis was made from samples taken at mediastinoscopy (four patients) or thoracotomy (two patients) (table 2).

All 17 patients were successfully treated with a short course of standard antituberculous therapy.

\section{Discussion}

Although lymph node enlargement as a manifestation of primary tuberculosis is much more common in children, the presentation in adults of such an abnormality on a chest radiograph is not rare. ${ }^{34}$ In a large series reported in 1959 by Lyons and coworkers ${ }^{5}$ tuberculosis was the fifth commonest cause of mediastinal enlargement, accounting for $6 \%$ of 782 cases. Intrathoracic lymphadenitis has been found to be present in between $0.5 \%$ and $26 \%$ of cases of tuberculosis in earlier studies. ${ }^{26-8}$ Our lower incidence of intrathoracic lymphadenitis at $0.25 \%$ of cases may be due to our selection of only patients who did not have any demonstrable parenchymal lung lesion, which was not applied in previous studies. We found right paratracheal lymphadenopathy in all our patients with intrathoracic lymphadenitis and this has been found to be the most commonly affected site in other studies. ${ }^{23}$

The diagnostic value of sputum examination is low in patients without parenchymal lesions of tuberculosis. The value of bronchoscopy in this situation has not been extensively evaluated and Cameron $e t a l^{9}$ noted that the procedure had no diagnostic merit under these circumstances. Chang and coworkers ${ }^{2}$ observed 
endobronchial involvement in 12 out of 16 patients $(75 \%)$ with intrathoracic tuberculous lymphadenopathy who underwent bronchoscopy. In our study bronchoscopy yielded the diagnosis in only nine out of 17 patients $(53 \%)$. This lower success may be explained by the absence of parenchymal disease in all our patients. We found a very high diagnostic yield from mucosal biopsy samples taken from the site of ulcerating granuloma and a $45 \%$ success rate from transbronchial or transcarinal aspiration in the presence of tracheal, carinal, or bronchial displacement by enlarged lymph nodes. This high yield may relate to our use of wide bore needles with the rigid bronchoscope.

The presence of mediastinal lymphadenopathy, a positive PPD skin test, and a chest radiograph suggesting a parenchymal lesion are all highly suggestive of the diagnosis of intrathoracic lymphadenitis. However, the absence of any parenchymal lesions and a high population prevalence of PPD skin positivity, such as is found in Turkey, make a definitive diagnosis of intrathoracic lymphadenitis difficult. Our study indicated that rigid bronchoscopy with appropriate sampling can give the diagnosis in about half of such cases, and direct lymph node biopsy, which may require mediastinoscopy or thoracotomy, diagnosed the remaining patients.

1 Wallace J, Deutsch A, Harrell J, Moser K. Bronchoscopy and transbronchial biopsy in evaluation of patients with suspected active tuberculosis. Am f Med 1981;70:1189-94.

2 Chang SC, Lee PY, Perng RP. Clinical role of bronchoscopy in adults with intrathoracic tuberculous lymphadenopathy.

3 Amorosa JK, Smith PR, Cohen JR, Ramsey C, Lyons HA. Tuberculous mediastinal lymphadenitis in adults. Radiology 1978;126:365-8.

4 Woodring JH, Vandiviere HM, Fried AM, Dillon M, Williams TD, Melvin IG. Update: the radiographic features of pulmonary tuberculosis. $A f R$ 1986;146:497-506.

5 Lyons HA, Calvy GL, Sammons BP. The diagnosis and classification of mediastinal masses. A study of 782 cases. Ann Intern Med 1959;51:897-932.

6 Choyke PL, Sostman HD, Curtis AM, Ravin CE, Chen JTT, Godwin JD, et al. Adult-onset pulmonary tuberculosis. Radiology 1983;148:357-62.

7 Bloomberg TJ, Dow CJ. Contemporary mediastinal tuberculosis. Thorax 1980;35:392-6.

8 Hadlock FP, Park SK, Awe RJ, Rivera M. Unusual radiographic findings in adult pulmonary tuberculosis. $A f R$ graphic findings in

9 Cameron EWJ. Tuberculosis and mediastinoscopy. Thorax 1978;33:117-20.

\title{
Impairment of endothelium-dependent pulmonary vasodilation in patients with primary pulmonary hypertension
}

\author{
Stephen J Brett, J Simon R Gibbs, John R Pepper, Timothy W Evans
}

Unit of Critical Care $S$ J Brett

$\mathrm{T}$ W Evans

Department of

Cardiology

J S R Gibbs

Department of Cardiothoracic Surgery

J R Pepper

National Heart and Lung Institute and Royal Brompton

Hospital,

London, SW3 6NP, UK

Correspondence to: Dr T W Evans.

Received 14 March 1995 Returned to authors

19 August 1995

19 August 1995

8 September 1995

8 September 1995

12 September 1995
Background - Pulmonary vascular tone may be modulated by endotheliumderived vasoactive mediators. Endothelial dysfunction is thought to occur in primary pulmonary hypertension. The aim of this study was to evaluate the vascular responses of patients with severe primary pulmonary hypertension to endotheliumdependent vasodilators (for example, substance $P)$ and non-endotheliumdependent vasodilators (for example, adenosine).

Methods - Six patients with primary pulmonary hypertension (mean (SE) systolic, diastolic, and pulmonary artery pressures $91 \cdot 1(7), 45 \cdot 2(3)$, and $62(4 \cdot 2) \mathrm{mm} \mathrm{Hg}$, respectively, and baseline total pulmonary vascular resistance (TPVR) 1949 (164) dynes $\cdot s \cdot \mathbf{c m}^{-5}$ ) underwent sequential infusions of substance $P(5-100 \mathrm{pmol} / \mathrm{min})$ and adenosine $(5-50 \mu \mathrm{g} / \mathrm{kg} / \mathrm{min})$ in random order. Pulmonary and systemic haemodynamics were monitored by indwelling radial and pulmonary arterial catheters.

Results - Substance P caused a marked fall in systemic vascular resistance (SVR) but minimal pulmonary vasodilation (mean maximal percentage change from baseline in TPVR:SVR ratio $27 \cdot 85(6 \cdot 5) \%$, p <0.01). Adenosine caused TPVR to fall, but resulted in no change in SVR (mean maximum percentage change from baseline in TPVR:SVR ratio $-9.85(3.5) \%$, p $<0 \cdot 05)$.

Conclusion - Endothelium-dependent vasodilation is deficient in the pulmonary circulation of patients with primary pulmonary hypertension and may contribute to the abnormalities of pulmonary vascular tone and reactivity seen in that condition.

(Thorax 1996;51:89-91)

Keywords: endothelium, primary pulmonary hypertension.

Primary pulmonary hypertension is rare, occurring most frequently in women in their third and fourth decades, and with a mean survival time after onset of symptoms of only 2-3 years. ${ }^{1}$ Early studies speculated that vasoconstriction was a major component of the increase in pulmonary vascular resistance (PVR) that characterises the condition. However, more recently 